\title{
Real-time observation of ultrafast molecular rotation in weakly bound dimers
}

\author{
Jiaqi Zhou $\odot,{ }^{1,2}$ Chaoxiong He, ${ }^{3}$ Ming-Ming Liu, ${ }^{3}$ Enliang Wang $\odot,{ }^{2,4}$ Shaokui Jia, ${ }^{1}$ Alexander Dorn $\odot,{ }^{2}$ \\ Xueguang Ren $\odot,^{1,2, *}$ and Yunquan Liu ${ }^{3,5,6, \dagger}$ \\ ${ }^{1}$ MOE Key Laboratory for Nonequilibrium Synthesis and Modulation of Condensed Matter, School of Physics, Xi'an Jiaotong University, \\ Xi'an 710049, China \\ ${ }^{2}$ Max-Planck-Institut für Kernphysik, Saupfercheckweg 1, 69117 Heidelberg, Germany \\ ${ }^{3}$ State Key Laboratory for Mesoscopic Physics and School of Physics, Peking University, Beijing 100871, China \\ ${ }^{4}$ J. R. Macdonald Laboratory, Department of Physics, Kansas State University, Manhattan, Kansas 66506, USA \\ ${ }^{5}$ Collaborative Innovation Center of Quantum Matter, Beijing 100871, China \\ ${ }^{6}$ Collaborative Innovation Center of Extreme Optics, Shanxi University, Taiyuan, Shanxi 030006, China
}

(Received 14 September 2020; accepted 31 March 2021; published 16 April 2021)

\begin{abstract}
The fragmentation dynamics of dicationic dimers of acetylene molecules initiated upon strong-field laser ionization is studied. Time-resolved pump-probe experiments with femtosecond laser pulses, accompanied by $a b$ initio dynamical calculations, allow us to evaluate the detailed behavior of molecular ions during the dissociation process. The dynamical properties of the intermediate $\mathrm{C}_{2} \mathrm{H}_{2}^{+} \cdots \mathrm{C}_{2} \mathrm{H}_{2}^{+}$state created by the pump pulse are probed by a second pulse which causes further ionization. The time-dependent yield of a coincident $\mathrm{C}_{2} \mathrm{H}_{2}^{+}+\mathrm{C}_{2} \mathrm{H}_{2}{ }^{2+}$ ion pair exhibits an oscillation feature with a periodicity of $240 \pm 30 \mathrm{fs}$. Our studies demonstrate that this is caused by an ultrafast rotation of $\mathrm{C}_{2} \mathrm{H}_{2}^{+}$cations driven by intermolecular Coulomb force together with the orientation-dependent ionization rate. We suggest that the present observation of ultrafast molecular rotation can be a general phenomenon occurring in a wide variety of systems.
\end{abstract}

DOI: 10.1103/PhysRevResearch.3.023050

\section{INTRODUCTION}

Weak noncovalent or intermolecular interactions, such as hydrogen bonding and van der Waals forces, are significant in all realms of chemistry ranging from supramolecular sciences to chemical reactions [1-6]. In recent years, studying the properties of excited states in weakly bound systems has attracted considerable interest due to the possibility for opening various ultrafast energy and charge transfer processes like the intermolecular Coulombic decay (ICD) [7]. In ICD, the innervalence vacancy is filled by an outer shell electron, and the energy released is transferred to neighboring molecules. This leads to ejection of a low-energy electron and formation of two repulsive ions at a distance of a few angstroms (Coulomb explosion).

It has been shown that ICD is a very general phenomenon occurring after a manifold of excitation schemes and in numerous weakly bound systems (see, e.g., [8-10]). The importance of ICD as an efficient source of low-energy electrons has been widely recognized, both for fundamental reasons

\footnotetext{
*renxueguang@xjtu.edu.cn

†yunquan.liu@pku.edu.cn
}

Published by the American Physical Society under the terms of the Creative Commons Attribution 4.0 International license. Further distribution of this work must maintain attribution to the author(s) and the published article's title, journal citation, and DOI. Open access publication funded by the Max Planck Society. and because of its potential relevance for radiation damage of biological matter. A detailed understanding of the quantum behaviors of ionic molecular species following ICD remains unexpectedly incomplete despite the paramount importance of these radical ions as reactive intermediates in the chemical and biological sciences. Moreover, such repulsive ion species are also observed through various mechanisms in weakly bound systems such as electron transfer mediated decay [11-13], strong-field laser ionization [14-16], direct Coulomb explosion by ion collision [17,18], and so forth. A recent calculation by Vendrell et al. investigated the fragmentation dynamics of two neighboring $\mathrm{H}_{2} \mathrm{O}^{+}$ions, i.e., the dicationic state after ICD in a water dimer [19]. Interestingly, the calculation revealed a significant change in the rotational energy and angular momentum of these species, in particular within a propagation time of about $150 \mathrm{fs}$.

In this work, we perform time-resolved pump-probe experiments [20,21] in molecular dimers using strong-field femtosecond laser pulses [15] in order to trace the rotational as well as vibrational and translational dynamics of molecular species during Coulomb explosion. Here, we choose the dimer of simple organic acetylene molecules as a target system. The ionization rate of the acetylene cation $\mathrm{C}_{2} \mathrm{H}_{2}{ }^{+}$is sensitively dependent on the orientation of molecules relative to the laser polarization direction of the probe pulse [22,23], which can be used to study the rotational properties of molecular ions.

As illustrated in Fig. 1(a), the neutral dimer is directly populated to a repulsive $\mathrm{C}_{2} \mathrm{H}_{2}+\ldots \mathrm{C}_{2} \mathrm{H}_{2}{ }^{+}$state upon multiphoton absorption. A second probe pulse causes further ionization of the system onto a more repulsive $\mathrm{C}_{2} \mathrm{H}_{2}+\ldots \mathrm{C}_{2} \mathrm{H}_{2}{ }^{2+}$ 

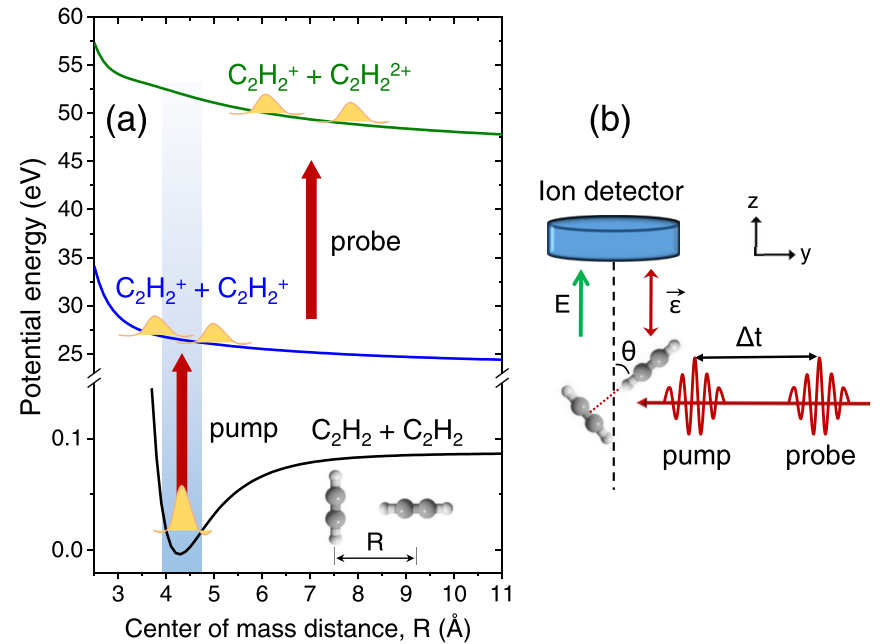

FIG. 1. (a) Illustration of the pump-probe experiment and the relevant potential energy curves for the neutral and ionized states of $\left(\mathrm{C}_{2} \mathrm{H}_{2}\right)_{2}$ calculated using the second-order Møller-Plesset perturbation theory with the aug-cc-pVTZ basis set. (b) Schematic view of the experimental geometry. The green arrow denotes the direction of the extracted electric field; $\vec{\varepsilon}$ is the laser polarization direction $(z$ direction).

tricationic dimer. We measured the kinetic energy release (KER) of the $\mathrm{C}_{2} \mathrm{H}_{2}{ }^{+}+\mathrm{C}_{2} \mathrm{H}_{2}{ }^{2+}$ Coulomb explosion as a function of time delay between the pump and probe pulses. This tracks the dynamical properties of the initially created $\mathrm{C}_{2} \mathrm{H}_{2}+\ldots \mathrm{C}_{2} \mathrm{H}_{2}{ }^{+}$state. Furthermore, we observed an oscillation of the time-dependent yield of $\mathrm{C}_{2} \mathrm{H}_{2}{ }^{+}+\mathrm{C}_{2} \mathrm{H}_{2}{ }^{2+}$ ion pairs with a periodicity of $240 \pm 30$ fs. This can be explained by an ultrafast rotation of $\mathrm{C}_{2} \mathrm{H}_{2}{ }^{+}$cations driven by intermolecular Coulomb forces, which is supported by our $a b$ initio molecular dynamics (AIMD) simulations. Our study implies that the present observation of molecular rotation is a general phenomenon in the dicationic complexes with two charges located in neighboring molecules. Indeed, our calculations indicate that it may also occur in the dimer of nitrogen molecules.

\section{EXPERIMENTAL METHODS}

As schematically shown in Fig. 1(b), the experiment was performed using a multiparticle imaging spectrometer which allows measuring the three-dimensional momentum vectors of ions and electrons in coincidence [15,24,25]. The acetylene dimer was generated by a supersonic expansion of a mixed gas of $\mathrm{Ar}$ and $\mathrm{C}_{2} \mathrm{H}_{2}(20 \%)$ through a 30- $\mu$ m nozzle into a vacuum chamber. This mixture obtains a branching ratio of about $4.5 \%$ for the $\left(\mathrm{C}_{2} \mathrm{H}_{2}\right)_{2}$ dimer in comparison with its monomer. The linearly polarized femtosecond laser pulse centered at $800 \mathrm{~nm}$ with a temporal duration of $25 \mathrm{fs}$ was generated from an amplified Ti:sapphire laser system. The laser beam was split into two halves with a continuous time delay at a resolution better than $3.5 \mathrm{fs}$ and focused to the center of the gas jet using a spherical mirror with a 75$\mathrm{mm}$ focal length. The peak density of the pump and probe pulses was estimated to be $I_{1}=I_{2} \simeq 2.0 \times 10^{14} \mathrm{~W} / \mathrm{cm}^{2}$ with the same polarization direction ( $z$ direction). The created
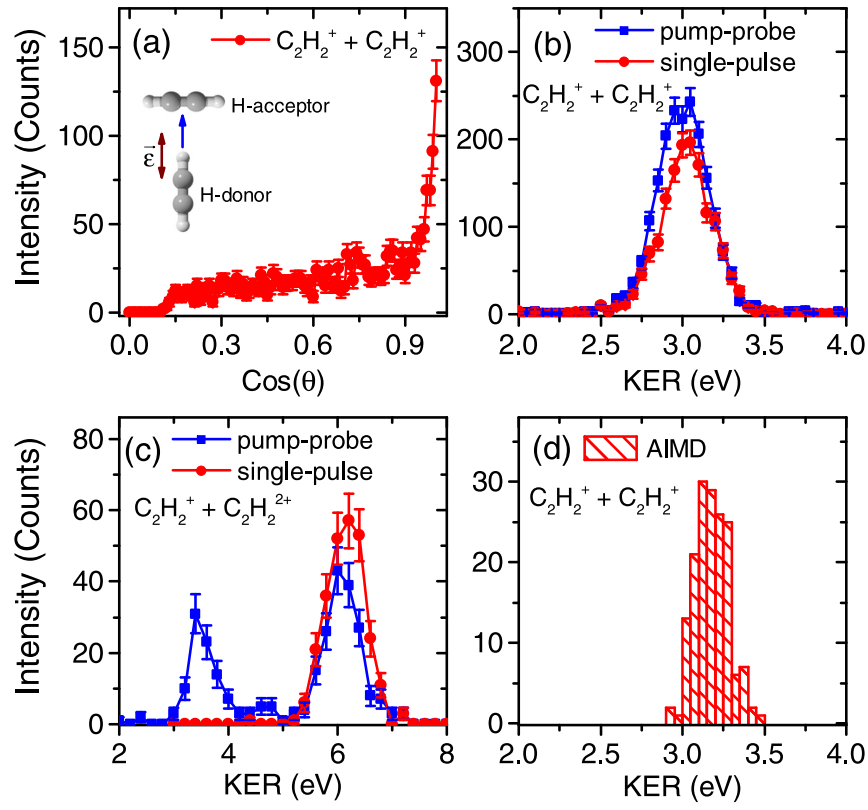

FIG. 2. (a) Measured ion emission angular distribution for the $\mathrm{C}_{2} \mathrm{H}_{2}{ }^{+}+\mathrm{C}_{2} \mathrm{H}_{2}{ }^{+}$channel. $\theta$ denotes the $\mathrm{C}_{2} \mathrm{H}_{2}{ }^{+}$emission angle (and thus the orientation of the intermolecular axis) relative to the laser polarization direction, as illustrated in Fig. 1(b). The inset shows the dimer configuration ( $\mathrm{T}$ shape) with dipole moment (blue arrow). (b) and (c) Single-pulse and pump-probe measured kinetic energy release (KER) distributions for the $\mathrm{C}_{2} \mathrm{H}_{2}{ }^{+}+\mathrm{C}_{2} \mathrm{H}_{2}{ }^{+}$and $\mathrm{C}_{2} \mathrm{H}_{2}{ }^{+}$ $+\mathrm{C}_{2} \mathrm{H}_{2}{ }^{2+}$ channels, respectively. (d) Ab initio molecular dynamics (AIMD) calculation of the KER for the $\mathrm{C}_{2} \mathrm{H}_{2}{ }^{+}+\mathrm{C}_{2} \mathrm{H}_{2}{ }^{+}$ion pair.

ion pairs, emerging from the interaction of laser pulses with dimers, were accelerated by a homogeneous electric field onto a multihit time- and position-sensitive detector, which allows us to reconstruct their momentum vectors and, consequently, the kinetic energies. We performed both pump-probe and single-pulse experiments for comparison.

\section{THEORETICAL CALCULATIONS}

We performed calculations for the Coulomb explosion channel of $\mathrm{C}_{2} \mathrm{H}_{2}^{+}+\mathrm{C}_{2} \mathrm{H}_{2}{ }^{+}$using AIMD simulations. In these calculations we considered an optimized $\mathrm{T}$-shaped dimer corresponding to the global-energy minimum geometry [see the inset in Fig. 2(a)]. The AIMD simulations were performed in two steps: (i) The initial conditions, i.e., geometries and velocities of every atom, of neutral $\left(\mathrm{C}_{2} \mathrm{H}_{2}\right)_{2}$ were sampled by the quasiclassical fixed normal-mode sampling method [26] under a temperature of $10 \mathrm{~K}$ in which the populations of the initial vibrational states were determined by Boltzmann distributions. (ii) We consider a vertical transition to the electronic ground state of the doubly charged $\left(\mathrm{C}_{2} \mathrm{H}_{2}{ }^{+} \ldots \mathrm{C}_{2} \mathrm{H}_{2}{ }^{+}\right)$dimer by the removal of an outermost $1 \pi_{u}$ electron from each of the $\mathrm{C}_{2} \mathrm{H}_{2}$ molecules. The dynamical simulations were performed under the extended Lagrangian molecular dynamics scheme adopting the so-called atomcentered density matrix propagation (ADMP) method [26-28] using the $\omega \mathrm{B} 97 \mathrm{XD}$ method that is a long-range corrected hybrid density-functional theory with the cc-pVTZ basis set. In order to obtain a well adiabatic control, we run the ADMP 
simulation with the keyword FULLSCF option, which means a converged self-consistent field simulation at each step. This is an effective way to maintain the dynamics on a given electronic state, i.e., equivalent to the Born-Oppenheimer surface [26-28]. Propagation was performed for $1000 \mathrm{fs}$ when an intermolecular distance of about $63.5 \AA$ was reached. The total KER can be obtained as the sum of the center-of-mass energies at this instant plus the remaining Coulomb energy. Overall, we consider the three-dimensional (both intramolecular and intermolecular) motions of the system as a function of propagation time for both the initial condition sampling of the neutral dimers and the dynamical simulations of the dicationic dimers. All calculations were carried out with the GAUSSIAN 16 suite of programs [29].

\section{RESULTS AND DISCUSSION}

Upon interaction with the pump pulse, the repulsive dicationic state $\left(\mathrm{C}_{2} \mathrm{H}_{2}{ }^{+} \ldots \mathrm{C}_{2} \mathrm{H}_{2}{ }^{+}\right)$is created, where the intermolecular hydrogen bond is oriented preferentially along the laser polarization vector $\vec{\varepsilon}$, as shown in the inset in Fig. 2(a). Since the $\mathrm{C}_{2} \mathrm{H}_{2}{ }^{+}+\mathrm{C}_{2} \mathrm{H}_{2}{ }^{+}$Coulomb explosion is faster than the rotation of the dimer system, we determine experimentally the orientation of the intermolecular hydrogen bond by the emission direction of $\mathrm{C}_{2} \mathrm{H}_{2}{ }^{+}$ionic fragments based on the axial recoil approximation [30]. Here, the fragmenting double ionization of $\left(\mathrm{C}_{2} \mathrm{H}_{2}\right)_{2}$ with hydrogen-bond breaking is favored when the intermolecular axis is oriented along the laser polarization direction [31]. This is confirmed by the angular distribution presented in Fig. 2(a), where a narrow angular distribution along the laser polarization is observed. The tendency of the $\mathrm{C}_{2} \mathrm{H}_{2}{ }^{+} \ldots \mathrm{C}_{2} \mathrm{H}_{2}{ }^{+}$state to dissociate along the polarization direction allows us to trace the motions of ionic species during the Coulomb explosion. These results will be discussed in the following sections.

The measured KERs for the Coulomb explosion channels of $\mathrm{C}_{2} \mathrm{H}_{2}{ }^{+}+\mathrm{C}_{2} \mathrm{H}_{2}{ }^{+}$and $\mathrm{C}_{2} \mathrm{H}_{2}^{+}+\mathrm{C}_{2} \mathrm{H}_{2}^{2+}$ are presented in Figs. 2(b) and 2(c), respectively, performed with both single-pulse and pump-probe experiments. For the latter data all pump-probe delays (50-1000 fs) were summed. For the $\mathrm{C}_{2} \mathrm{H}_{2}{ }^{+}+\mathrm{C}_{2} \mathrm{H}_{2}{ }^{+}$ion pair, the KER distributions are almost identical for both experiments, which show a single peak located at KER $\sim 3.05 \mathrm{eV}$. The AIMD calculated KER distribution is presented in Fig. 2(d) with a peak value of about $3.18 \mathrm{eV}$. This is in rather good agreement with the experimental KER, while the slight deviations in peak values can arise from the deformation of the dimer structure induced by strong laser fields in the experiments [32]. We notice that the calculated KER is in excellent agreement with the coincident measurements of the $\mathrm{C}_{2} \mathrm{H}_{2}{ }^{+}+\mathrm{C}_{2} \mathrm{H}_{2}{ }^{+}$ion pair induced by ion collisions [33].

For the $\mathrm{C}_{2} \mathrm{H}_{2}{ }^{+}+\mathrm{C}_{2} \mathrm{H}_{2}{ }^{2+}$ ion pair in Fig. 2(c), however, we observed different KER distributions between single-pulse and pump-probe experiments. The single-pulse measured KER shows a unimodal structure with a peak value at $\sim 6.1 \mathrm{eV}$, while the KER obtained in the pump-probe experiment exhibits an additional peak located at $\sim 3.4 \mathrm{eV}$. The peak at KER $\sim 6.1 \mathrm{eV}$ can be understood by the direct population of the dimer from its neutral ground state to the repulsive tricationic state $\left(\mathrm{C}_{2} \mathrm{H}_{2}{ }^{+} \ldots \mathrm{C}_{2} \mathrm{H}_{2}{ }^{2+}\right)$ induced by a single pulse.
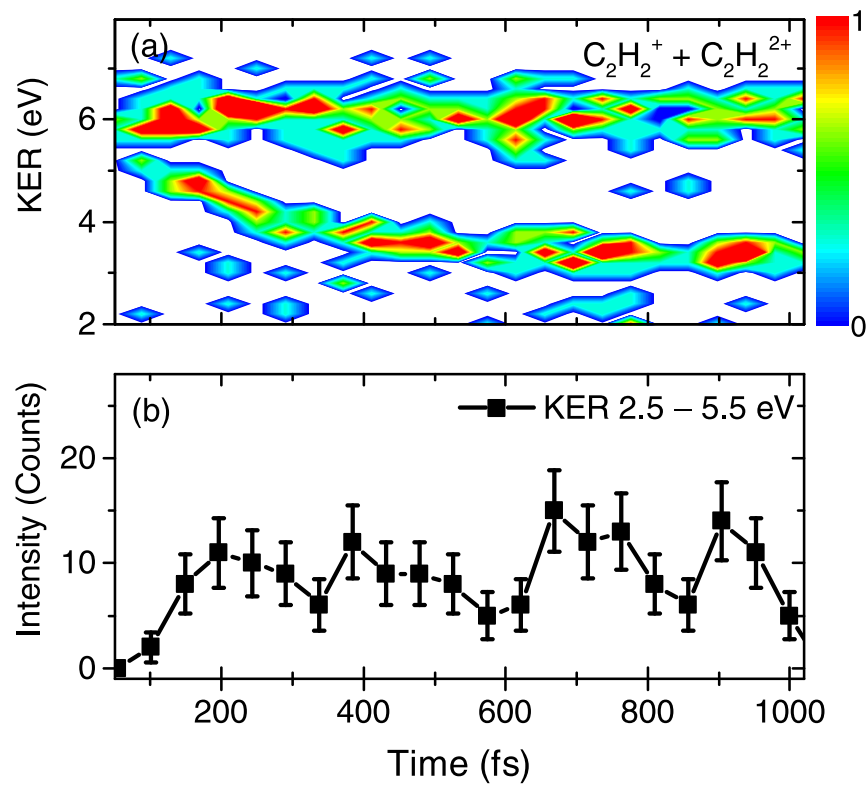

FIG. 3. (a) Measured KER of the coincident $\mathrm{C}_{2} \mathrm{H}_{2}{ }^{+}+\mathrm{C}_{2} \mathrm{H}_{2}{ }^{2+}$ ion pair versus the time delay spectrum. (b) Projection of KERs between 2.5 and $5.5 \mathrm{eV}$ on the time axis.

This matches well with the value of about $6.5 \mathrm{eV}$ estimated by calculating the kinetic energy of two point charges starting a Coulomb explosion at the mean intermolecular distance of the $\left(\mathrm{C}_{2} \mathrm{H}_{2}\right)_{2}$ dimer (4.4 $\AA$ ) [34-36]. The additional peak (KER $\sim 3.4 \mathrm{eV}$ ) arises from stepwise ionization by the pump and the delayed probe pulses. Thus, by continuously scanning the time delay and recording the KER of the corresponding ion pair, the wave packet motion of intermediate states can be traced in real time $[21,37]$.

In Fig. 3(a) the measured KER of the coincident $\mathrm{C}_{2} \mathrm{H}_{2}{ }^{+}+$ $\mathrm{C}_{2} \mathrm{H}_{2}{ }^{2+}$ ion pair is plotted as a function of delay time. This spectrum shows two different features: one is time dependent, with decreasing KER towards larger time delay, and the other one is time-independent contributions for KER around $6.1 \mathrm{eV}$. As mentioned above, the latter is a result of a direct process via a single (pump/probe) pulse. However, the timedependent component starts from about $6.1 \mathrm{eV}$, decreases as time increases, and asymptotically reaches $3.4 \mathrm{eV}$ energy, which are clear signatures of delayed ionization.

There are three possible intermediate states, i.e., $\mathrm{C}_{2} \mathrm{H}_{2}{ }^{+} \ldots \mathrm{C}_{2} \mathrm{H}_{2}, \mathrm{C}_{2} \mathrm{H}_{2}{ }^{2+} \ldots \mathrm{C}_{2} \mathrm{H}_{2}$, and $\mathrm{C}_{2} \mathrm{H}_{2}{ }^{+} \ldots \mathrm{C}_{2} \mathrm{H}_{2}{ }^{+}$. However, only one state is initiated in the present experiment. We can determine $\mathrm{C}_{2} \mathrm{H}_{2}{ }^{+} \ldots \mathrm{C}_{2} \mathrm{H}_{2}{ }^{+}$is the relevant intermediate state by comparing the asymptotic energies in the time-dependent KER spectrum with the various dissociation channels $[37,38]$. The pump pulse removes one electron from the outermost $\left(1 \pi_{u}\right)$ orbital in each molecule of the dimer to reach this dicationic state. Subsequently, two $\mathrm{C}_{2} \mathrm{H}_{2}{ }^{+}$ions propagate freely on the potential energy curve of the intermediate state until the probe pulse arrives. Then, one of the $\mathrm{C}_{2} \mathrm{H}_{2}{ }^{+}$is further ionized and projected to the final $\mathrm{C}_{2} \mathrm{H}_{2}+\ldots \mathrm{C}_{2} \mathrm{H}_{2}{ }^{2+}$ state. During the dissociation, the wave packet moves towards a larger intermolecular distance under the action of Coulomb force, resulting in the decrease of KER. 
Moreover, the spectrum exhibits an oscillation structure in the yield of the ion pair. This feature is more evident in Fig. 3(b), where the ion yield for KER between 2.5 and $5.5 \mathrm{eV}$ is projected onto the time axis. We found an oscillation with a period of $240 \pm 30 \mathrm{fs}$. First, this oscillation is statistically significant, which can be seen by comparing it with the ion pair yield for the KER region between 5.5 and $6.5 \mathrm{eV}$ that shows no regular oscillations. Second, intramolecular vibration can be ruled out as the origin since experiments show a much shorter vibrational period of about 25 fs [39]. Third, intermolecular bond vibration can be excluded, causing periodically enhanced ionization since the pump pulse breaks the intermolecular hydrogen bond and initiates Coulomb explosion.

The observed oscillation is caused by an ultrafast rotation of $\mathrm{C}_{2} \mathrm{H}_{2}{ }^{+}$cations which is much faster than the rotational period of $14 \mathrm{ps}$ for neutral $\mathrm{C}_{2} \mathrm{H}_{2}$. The pump pulse creates $\mathrm{C}_{2} \mathrm{H}_{2}{ }^{+} \ldots \mathrm{C}_{2} \mathrm{H}_{2}{ }^{+}$oriented along the laser polarization axis. Here, one of the $\mathrm{C}_{2} \mathrm{H}_{2}{ }^{+}$cations in the intermediate state is further ionized via probe pulse to the nondissociative dication of $\mathrm{C}_{2} \mathrm{H}_{2}{ }^{2+}\left({ }^{3} \Sigma_{g}^{-},{ }^{1} \Delta_{g}\right.$, or $\left.{ }^{1} \Sigma_{g}^{+}\right)$in which two electrons are removed from the outermost orbital $[22,40]$. It has been shown that the strong-field ionization rate depends sensitively on the molecular orientation [22,23,31,41-43]. The $\mathrm{C}_{2} \mathrm{H}_{2}{ }^{+}$cation experiences an enhanced ionization rate $\left(\mathrm{C}_{2} \mathrm{H}_{2}{ }^{+} \rightarrow \mathrm{C}_{2} \mathrm{H}_{2}{ }^{2+}\right)$ when the cationic axis is perpendicular to the polarization direction because the outermost orbital is distributed perpendicular to the molecular axis [22,23]. The rotational angle of $\mathrm{C}_{2} \mathrm{H}_{2}{ }^{+}$is convoluted with the transition probability to the final state in the probe step. The yield of the $\mathrm{C}_{2} \mathrm{H}_{2}{ }^{+}+\mathrm{C}_{2} \mathrm{H}_{2}{ }^{2+}$ ion pair reaches maximum periodically whenever the probe pulse meets the rotating cation perpendicular to the polarization axis. This causes the oscillation in the time-dependent ion yield.

The present observations can be interpreted more quantitatively with the help of our AIMD simulations. Calculated molecular motions of the $\mathrm{C}_{2} \mathrm{H}_{2}{ }^{+}+\mathrm{C}_{2} \mathrm{H}_{2}{ }^{+}$Coulomb explosion are shown in Fig. 4(a), eventually forming the path-integral trajectories. It can be seen from Fig. 4(c) that after creating the dicationic dimer, ultrafast rotation is initiated in the $\mathrm{H}$-donor $\mathrm{C}_{2} \mathrm{H}_{2}{ }^{+} d$ cations as the rotational energy $E_{\text {rot }}$ of this ion has increased dramatically due to intermolecular Coulomb interaction. $E_{\text {rot }}$ converges to a value of $\sim 150 \mathrm{meV}$ at larger time, while for the $\mathrm{H}$-acceptor $\mathrm{C}_{2} \mathrm{H}_{2}{ }^{+} a$ ions, the obtained $E_{\text {rot }}$ is very small (about $1 \mathrm{meV}$ ) and almost constant over the whole range of delay time, as presented in Fig. 4(b). This means a much slower rotation of this ion, which is clearly visible from the rotational angle $\theta_{a}$ versus time spectrum presented in Fig. 4(d). Here, $\theta_{a}$ is the angle between the molecular orientation and the intermolecular axis that is also parallel to the laser polarization axis in the experiments.

As presented in Fig. 4(e), the rotational angle $\theta_{d}$ versus time spectrum reveals directly the ultrafast rotation of $\mathrm{C}_{2} \mathrm{H}_{2}{ }^{+}$ $d$ ions, from which an effective period for $2 \pi$ rotation is derived as $500 \pm 100 \mathrm{fs}$, corresponding to a rotational speed of about $2.0 \pm 0.5 \mathrm{THz}$. This ultrafast rotation causes the molecular orientation to be periodically perpendicular to the laser polarization [see Fig. 4(a)], where the cation experiences an enhanced rate for secondary ionization $\left(\mathrm{C}_{2} \mathrm{H}_{2}{ }^{+} \rightarrow \mathrm{C}_{2} \mathrm{H}_{2}{ }^{2+}\right)$. It will happen twice that the $\mathrm{C}_{2} \mathrm{H}_{2}^{+}$cation is perpendicular to the laser polarization within a $2 \pi$ rotational period, and thus,
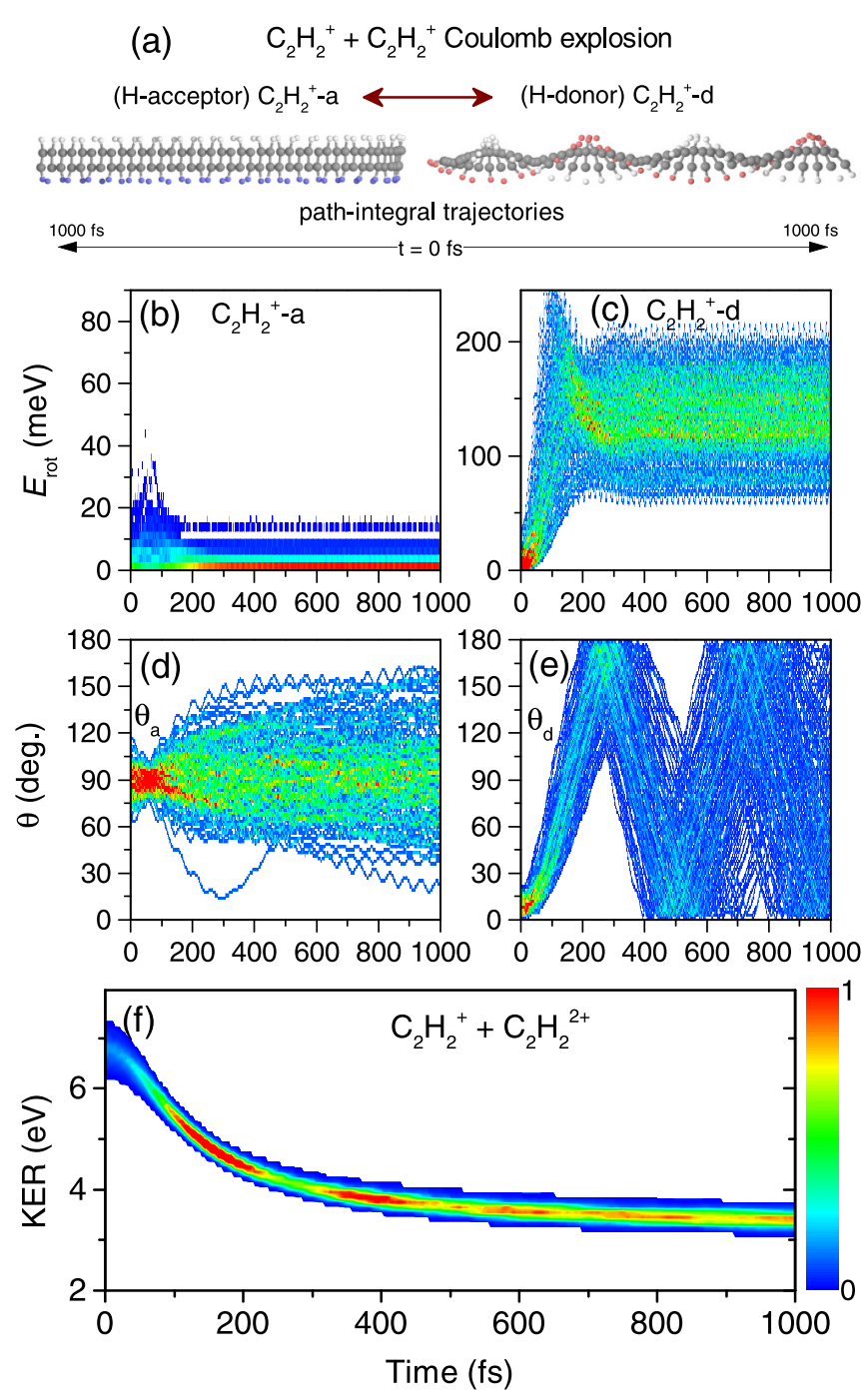

FIG. 4. (a) Calculated dynamics of two $\mathrm{C}_{2} \mathrm{H}_{2}{ }^{+}$ions under Coulomb explosion. The intermolecular axis is along the horizontal direction. Blue and red dots denote $\mathrm{H}$ atoms in two $\mathrm{C}_{2} \mathrm{H}_{2}{ }^{+}$cations for better visualization of molecular motions. (b) and (c) AIMD calculated rotational energies for the $\mathrm{H}$-acceptor $\mathrm{C}_{2} \mathrm{H}_{2}{ }^{+} a$ and $\mathrm{H}$-donor $\mathrm{C}_{2} \mathrm{H}_{2}{ }^{+} d$ ions, respectively. (d) and (e) AIMD calculated rotational angles of $\theta_{a}$ and $\theta_{d}$ relative to the intermolecular axis for $\mathrm{C}_{2} \mathrm{H}_{2}{ }^{+} a$ and $\mathrm{C}_{2} \mathrm{H}_{2}{ }^{+} d$ ions, respectively. (f) Calculated time-dependent KER spectrum of the $\mathrm{C}_{2} \mathrm{H}_{2}{ }^{+}+\mathrm{C}_{2} \mathrm{H}_{2}{ }^{2+}$ Coulomb explosion.

leads to the observed oscillation period of $240 \pm 30 \mathrm{fs}$ in the ion yield. The difference between experiment and simulation may result from the influence of the laser field, which is quantified by the so-called kick strength parameter using the delta-kick method $[44,45]$. In the present experiment, the maximum amount of angular momentum transferred from the laser pulse to the $\mathrm{C}_{2} \mathrm{H}_{2}$ molecule is determined to be $15.35 \hbar$, corresponding to a rotational energy of about $32.5 \mathrm{meV}$.

In addition, the calculated KER versus time spectrum is presented in Fig. 4(f). This spectrum is achieved by adding the Coulomb energy from secondary ionization to the real-time kinetic energies of two $\mathrm{C}_{2} \mathrm{H}_{2}{ }^{+}$cations. Additionally, we account for the orientation-dependent ionization rate $[22,23]$ by simulating the angular distribution for the second ionization 
process with the rotational angle $\theta_{d}$. It can be seen that the calculated spectrum is in very good agreement with the experimental result concerning both the time-dependent KER for the dissociation path and the ion yield oscillation in general. This confirms the present observation of ultrafast molecular rotation driven by intermolecular Coulomb force.

\section{CONCLUSIONS}

In summary, we performed time-resolved experiments using strong-field femtosecond laser pulses to investigate the molecular dynamics in the dicationic acetylene dimer with two charges located separately. After creating a repulsive $\mathrm{C}_{2} \mathrm{H}_{2}{ }^{+} \ldots \mathrm{C}_{2} \mathrm{H}_{2}{ }^{+}$state with the pump pulse, the time evolution of this state is detected by the probe pulse, which causes further ionization of the system to $\mathrm{C}_{2} \mathrm{H}_{2}{ }^{+} \cdots \mathrm{C}_{2} \mathrm{H}_{2}{ }^{2+}$. The dissociative motion of the ionic fragments is observed by the measurements of kinetic energy release of coincident $\mathrm{C}_{2} \mathrm{H}_{2}{ }^{+}+\mathrm{C}_{2} \mathrm{H}_{2}{ }^{2+}$ ion pairs as a function of the time delay between pump and probe pulses. Importantly, we observed an oscillation in the time-dependent yield of ion pairs. Our study indicates that after removing an outermost $1 \pi_{u}$ electron from each molecule, the $\mathrm{C}_{2} \mathrm{H}_{2}{ }^{+}$cations are subjected to intermolecular Coulomb force, leading to ultrafast rotation with a period time of $480 \pm 60 \mathrm{fs}$. This causes the $\mathrm{C}_{2} \mathrm{H}_{2}{ }^{+}$ion to be oriented perpendicular to the laser polarization two times within a $2 \pi$ rotation period where the cation experiences an enhanced rate for the second ionization and thus results in oscillation in the ion yield. These results, supported by our ab initio molecular dynamics calculations, reveal a previously unexpected phenomenon of ultrafast rotation in molecular dimers.

Our further calculations on $\left(\mathrm{N}_{2}\right)_{2}$ dimers, shown in Fig. 5, indicate that a similar feature appears also in the molecular nitrogen dimer. Here, the calculated KER for the $\mathrm{N}_{2}{ }^{+}+$ $\mathrm{N}_{2}{ }^{+}$ion pair is in good agreement with the measurement of ICD in $\left(\mathrm{N}_{2}\right)_{2}$ [46]. These results demonstrate that the present observation of ultrafast molecular rotation could be a general phenomenon occurring in a wide variety of systems. Our studies could have important implications in broad areas both for fundamental reasons of the molecular angular momentum and because of its role in many chemical, physical, and optical applications [47], e.g., the molecular rotation effects in hot-air lasing [48-50], the understanding of the Coulomb explosion
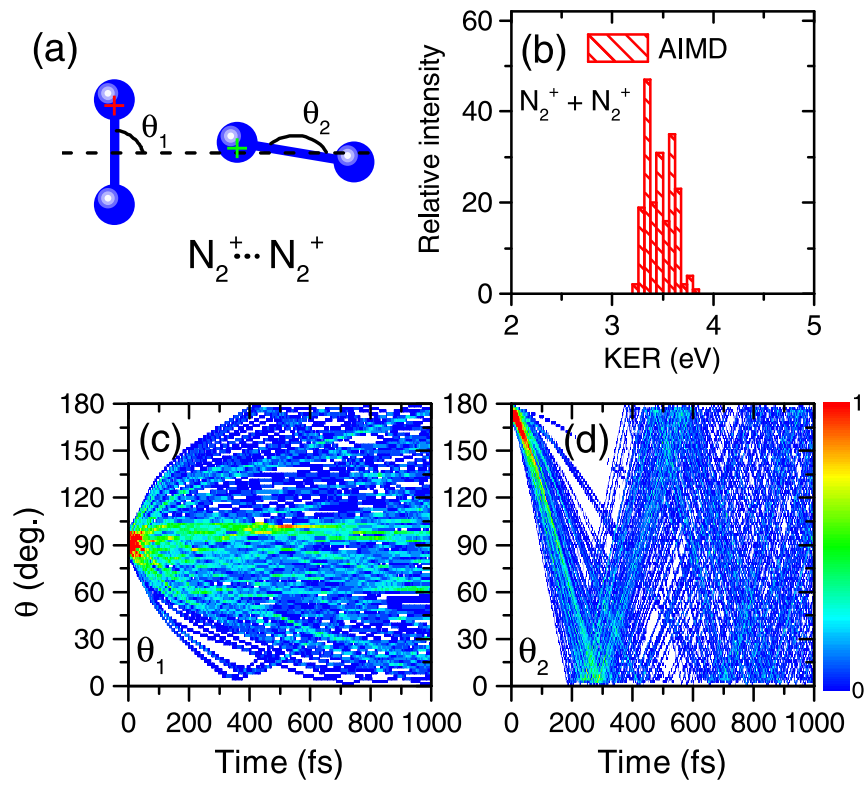

FIG. 5. (a) Dicationic dimer of molecular nitrogen. (b) AIMD calculated KER for the $\mathrm{N}_{2}{ }^{+}+\mathrm{N}_{2}{ }^{+}$ion pair. (c) and (d) AIMD calculated rotational angles relative to the intermolecular axis for $\theta_{1}$ (ion 1) and $\theta_{2}$ (ion 2), respectively, as described in (a).

imaging principle [51,52], and laser alignment of molecular clusters [53-55]. Furthermore, molecular reactivity strongly depends on the relative orientation of molecules with respect to one another. Therefore, understanding how an environment affects molecular rotations is crucial to control chemical reactions, in particular ion-molecule reactions $[3-6,56,57]$ and gas-surface interactions [58-60]. Future studies on more complex systems with higher signal-to-noise ratios are expected to provide more insights into the findings of this work.

\section{ACKNOWLEDGMENTS}

We thank R. Moshammer for valuable discussions. This work was jointly supported by the National Natural Science Foundation of China under Grants No. 11974272, No. 92050201, and No. 11774281 and by the Deutsche Forschungsgemeinschaft (DFG). J.Z. is grateful for support from the China Scholarship Council (CSC). E.W. acknowledges a fellowship from the Alexander von Humboldt Foundation.
[1] J. Řezáč and P. Hobza, Benchmark calculations of interaction energies in noncovalent complexes and their applications, Chem. Rev. 116, 5038 (2016).

[2] E. A. Meyer, R. K. Castellano, and F. Diederich, Interactions with aromatic rings in chemical and biological recognition, Angew. Chem., Int. Ed. 42, 1210 (2003).

[3] D. Hauser, S. Lee, F. Carelli, S. Spieler, O. Lakhmanskaya, E. S. Endres, S. S. Kumar, F. Gianturco, and R. Wester, Rotational state-changing cold collisions of hydroxyl ions with helium, Nat. Phys. 11, 467 (2015).
[4] Y. Shagam, A. Klein, W. Skomorowski, R. Yun, V. Averbukh, C. P. Koch, and E. Narevicius, Molecular hydrogen interacts more strongly when rotationally excited at low temperatures leading to faster reactions, Nat. Chem. 7, 921 (2015).

[5] W. E. Perreault, N. Mukherjee, and R. N. Zare, Quantum control of molecular collisions at 1 kelvin, Science 358, 356 (2017).

[6] A. Li, Y. Li, H. Guo, K.-C. Lau, Y. Xu, B. Xiong, Y.-C. Chang, and $\mathrm{C}$. Y. $\mathrm{Ng}$, Communication: The origin of rotational enhancement effect for the reaction of $\mathrm{H}_{2} \mathrm{O}^{+}+\mathrm{H}_{2}\left(\mathrm{D}_{2}\right)$, J. Chem. Phys. 140, 011102 (2014). 
[7] L. S. Cederbaum, J. Zobeley, and F. Tarantelli, Giant Intermolecular Decay and Fragmentation of Clusters, Phys. Rev. Lett. 79, 4778 (1997).

[8] R. Santra and L. S. Cederbaum, Non-Hermitian electronic theory and applications to clusters, Phys. Rep. 368, 1 (2002).

[9] U. Hergenhahn, Production of low kinetic energy electrons and energetic ion pairs by intermolecular Coulombic decay, Int. J. Radiat. Biol. 88, 871 (2012).

[10] T. Jahnke, U. Hergenhahn, B. Winter, R. Dörner, U. Frühling, P. V. Demekhin, K. Gokhberg, L. S. Cederbaum, A. Ehresmann, A. Knie, and A. Dreuw, Interatomic and intermolecular Coulombic decay, Chem. Rev. 120, 11295 (2020).

[11] I. Unger, R. Seidel, S. Thürmer, M. N. Pohl, E. F. Aziz, L. S. Cederbaum, E. Muchová, P. Slavíček, B. Winter, and N. V. Kryzhevoi, Observation of electron-transfer-mediated decay in aqueous solution, Nat. Chem. 9, 708 (2017).

[12] M. N. Pohl, C. Richter, E. Lugovoy, R. Seidel, P. Slavíček, E. F. Aziz, B. Abel, B. Winter, and U. Hergenhahn, Sensitivity of electron transfer mediated decay to ion pairing, J. Phys. Chem. B 121, 7709 (2017).

[13] V. Stumpf, K. Gokhberg, and L. S. Cederbaum, The role of metal ions in x-ray-induced photochemistry, Nat. Chem. 8, 237 (2016).

[14] B. Ulrich, A. Vredenborg, A. Malakzadeh, M. Meckel, K. Cole, M. Smolarski, Z. Chang, T. Jahnke, and R. Dörner, Doubleionization mechanisms of the argon dimer in intense laser fields, Phys. Rev. A 82, 013412 (2010).

[15] X. Xie, C. Wu, Z. Yuan, D. Ye, P. Wang, Y. Deng, L. Fu, J. Liu, Y. Liu, and Q. Gong, Dynamical coupling of electrons and nuclei for Coulomb explosion of argon trimers in intense laser fields, Phys. Rev. A 92, 023417 (2015).

[16] U. Saalmann, C. Siedschlag, and J. M. Rost, Mechanisms of cluster ionization in strong laser pulses, J. Phys. B 39, R39 (2006).

[17] J. Matsumoto, A. Leredde, X. Flechard, K. Hayakawa, H. Shiromaru, J. Rangama, C. L. Zhou, S. Guillous, D. Hennecart, T. Muranaka, A. Mery, B. Gervais, and A. Cassimi, Asymmetry in Multiple-Electron Capture Revealed by Radiative Charge Transfer in Ar Dimers, Phys. Rev. Lett. 105, 263202 (2010).

[18] H.-K. Kim, H. Gassert, J. N. Titze, M. Waitz, J. Voigtsberger, F. Trinter, J. Becht, A. Kalinin, N. Neumann, C. Zhou, L. P. H. Schmidt, O. Jagutzki, A. Czasch, M. Schöffler, H. Merabet, H. Schmidt-Böcking, T. Jahnke, H. J. Lüdde, A. Cassimi, and R. Dörner, Orientation dependence in multiple ionization of $\mathrm{He}_{2}$ and $\mathrm{Ne}_{2}$ induced by fast, highly charged ions: Probing the impact-parameter-dependent ionization probability in 11.37$\mathrm{MeV} / \mathrm{u} \mathrm{S}^{14+}$ collisions with He and Ne, Phys. Rev. A 89, 022704 (2014).

[19] O. Vendrell, S. D. Stoychev, and L. S. Cederbaum, Generation of highly damaging $\mathrm{H}_{2} \mathrm{O}^{+}$radicals by inner valence shell ionization of water, ChemPhysChem 11, 1006 (2010).

[20] A. H. Zewail, Femtochemistry: Atomic-scale dynamics of the chemical bond, J. Phys. Chem. A 104, 5660 (2000).

[21] T. Ergler, A. Rudenko, B. Feuerstein, K. Zrost, C. D. Schröter, R. Moshammer, and J. Ullrich, Spatiotemporal Imaging of Ultrafast Molecular Motion: Collapse and Revival of the $\mathrm{D}_{2}{ }^{+}$ nuclear wave packet, Phys. Rev. Lett. 97, 193001 (2006).

[22] K. Doblhoff-Dier, M. Kitzler, and S. Gräfe, Theoretical investigation of alignment-dependent intense-field fragmentation of acetylene, Phys. Rev. A 94, 013405 (2016).

[23] X. Xie, K. Doblhoff-Dier, H. Xu, S. Roither, M. S. Schöffler, D. Kartashov, S. Erattupuzha, T. Rathje, G. G. Paulus, K. Yamanouchi, A. Baltuška, S. Gräfe, and M. Kitzler, Selective Control over Fragmentation Reactions in Polyatomic Molecules Using Impulsive Laser Alignment, Phys. Rev. Lett. 112, 163003 (2014).

[24] J. Ullrich, R. Moshammer, A. Dorn, R. Dörner, L. Schmidt, and H. Schmidt-Böcking, Recoil-ion and electron momentum spectroscopy: Reaction-microscopes, Rep. Prog. Phys. 66, 1463 (2003).

[25] R. Dörner, V. Mergel, O. Jagutzki, L. Spielberger, J. Ullrich, R. Moshammer, and H. Schmidt-Böcking, Cold target recoil ion momentum spectroscopy: A 'momentum microscope' to view atomic collision dynamics, Phys. Rep. 330, 95 (2000).

[26] H. B. Schlegel, J. M. Millam, S. S. Iyengar, G. A. Voth, A. D. Daniels, G. E. Scuseria, and M. J. Frisch, Ab initio molecular dynamics: Propagating the density matrix with gaussian orbitals, J. Chem. Phys. 114, 9758 (2001).

[27] S. S. Iyengar, H. B. Schlegel, J. M. Millam, G. A. Voth, G. E. Scuseria, and M. J. Frisch, Ab initio molecular dynamics: Propagating the density matrix with Gaussian orbitals. II. Generalizations based on mass-weighting, idempotency, energy conservation and choice of initial conditions, J. Chem. Phys. 115, 10291 (2001).

[28] H. B. Schlegel, S. S. Iyengar, X. Li, J. M. Millam, G. A. Voth, G. E. Scuseria, and M. J. Frisch, Ab initio molecular dynamics: Propagating the density matrix with gaussian orbitals. III. Comparison with Born-Oppenheimer dynamics, J. Chem. Phys. 117, 8694 (2002).

[29] M. J. Frisch et al., GAUSSIAN 16, revision A.03, Gaussian Inc., Wallingford, CT, 2016.

[30] R. N. Zare, Dissociation of $\mathrm{H}_{2}^{+}$by electron impact: Calculated angular distribution, J. Chem. Phys. 47, 204 (1967).

[31] J. Wu, X. Gong, M. Kunitski, F. K. Amankona-Diawuo, L. P. H. Schmidt, T. Jahnke, A. Czasch, T. Seideman, and R. Dörner, Strong Field Multiple Ionization as a Route to Electron Dynamics in a van der Waals Cluster, Phys. Rev. Lett. 111, 083003 (2013).

[32] A. Hishikawa, A. Iwamae, and K. Yamanouchi, Ultrafast Deformation of the Geometrical Structure of $\mathrm{CO}_{2}$ Induced in Intense Laser Fields, Phys. Rev. Lett. 83, 1127 (1999).

[33] S. Xu, D. Guo, X. Ma, X. Zhu, W. Feng, S. Yan, D. Zhao, Y. Gao, S. Zhang, X. Ren, Y. Zhao, Z. Xu, A. Dorn, L. S. Cederbaum, and N. V. Kryzhevoi, Damaging intermolecular energy and proton transfer processes in alpha-particle-irradiated hydrogen-bonded systems, Angew. Chem., Int. Ed. 57, 17023 (2018).

[34] A. Karpfen, The dimer of acetylene and the dimer of diacetylene: A floppy and a very floppy molecule, J. Phys. Chem. A 103, 11431 (1999).

[35] C. Leforestier, A. Tekin, G. Jansen, and M. Herman, First principles potential for the acetylene dimer and refinement by fitting to experiments, J. Chem. Phys. 135, 234306 (2011).

[36] K. Shuler and C. E. Dykstra, Interaction potentials and vibrational effects in the acetylene dimer, J. Phys. Chem. A 104, 4562 (2000).

[37] I. A. Bocharova, A. S. Alnaser, U. Thumm, T. Niederhausen, D. Ray, C. L. Cocke, and I. V. Litvinyuk, Time-resolved Coulomb- 
explosion imaging of nuclear wave-packet dynamics induced in diatomic molecules by intense few-cycle laser pulses, Phys. Rev. A 83, 013417 (2011).

[38] K. Schnorr et al., Time-Resolved Measurement of Interatomic Coulombic Decay in $\mathrm{Ne}_{2}$, Phys. Rev. Lett. 111, 093402 (2013).

[39] C. Burger, A. Atia-Tul-Noor, T. Schnappinger, H. Xu, P. Rosenberger, N. Haram, S. Beaulieu, F. Légaré, A. S. Alnaser, R. Moshammer, R. T. Sang, B. Bergues, M. S. Schuurman, R. de Vivie-Riedle, I. V. Litvinyuk, and M. F. Kling, Time-resolved nuclear dynamics in bound and dissociating acetylene, Struct. Dyn. 5, 044302 (2018)

[40] S. Andrews, F. Harris, and D. Parry, A combined experimental and theoretical investigation of $\mathrm{C}_{2} \mathrm{H}_{2}^{2+}$ electronic-state energies, Chem. Phys. 166, 69 (1992).

[41] G. L. Kamta and A. D. Bandrauk, Phase Dependence of Enhanced Ionization in Asymmetric Molecules, Phys. Rev. Lett. 94, 203003 (2005).

[42] E. Constant, H. Stapelfeldt, and P. B. Corkum, Observation of Enhanced Ionization of Molecular Ions in Intense Laser Fields, Phys. Rev. Lett. 76, 4140 (1996).

[43] T. Zuo and A. D. Bandrauk, Charge-resonance-enhanced ionization of diatomic molecular ions by intense lasers, Phys. Rev. A 52, R2511 (1995).

[44] Y. Khodorkovsky, U. Steinitz, J.-M. Hartmann, and I. S. Averbukh, Collisional dynamics in a gas of molecular superrotors, Nat. Commun. 6, 7791 (2015).

[45] C. Wu, G. Zeng, H. Jiang, Y. Gao, N. Xu, and Q. Gong, Molecular rotational excitation by strong femtosecond laser pulses, J. Phys. Chem. A 113, 10610 (2009).

[46] F. Trinter, M. S. Schöffler, H.-K. Kim, F. P. Sturm, K. Cole, N. Neumann, A. Vredenborg, J. Williams, I. Bocharova, R. Guillemin, M. Simon, A. Belkacem, A. L. Landers, T. Weber, H. Schmidt-Böcking, R. Dörner, and T. Jahnke, Resonant auger decay driving intermolecular Coulombic decay in molecular dimers, Nature (London) 505, 664 (2014).

[47] C. P. Koch, M. Lemeshko, and D. Sugny, Quantum control of molecular rotation, Rev. Mod. Phys. 91, 035005 (2019).

[48] M. Britton, M. Lytova, D. H. Ko, A. Alqasem, P. Peng, D. M. Villeneuve, C. Zhang, L. Arissian, and P. B. Corkum, Control of $\mathrm{N}_{2}{ }^{+}$air lasing, Phys. Rev. A 102, 053110 (2020).

[49] H. Zhang, C. Jing, J. Yao, G. Li, B. Zeng, W. Chu, J. Ni, H. Xie, H. Xu, S. L. Chin, K. Yamanouchi, Y. Cheng, and Z. Xu, Rotational Coherence Encoded in an "Air-Laser" Spectrum of Nitrogen Molecular Ions in an Intense Laser Field, Phys. Rev. X 3, 041009 (2013).
[50] T. Ando, E. Lötstedt, A. Iwasaki, H. Li, Y. Fu, S. Wang, H. $\mathrm{Xu}$, and $\mathrm{K}$. Yamanouchi, Rotational, Vibrational, and Electronic Modulations in $\mathrm{N}_{2}^{+}$Lasing at $391 \mathrm{~nm}$ : Evidence of Coherent $b^{2} \Sigma_{u}^{+}-x^{2} \Sigma_{g}^{+}-a^{2} \Pi_{u}$ Coupling, Phys. Rev. Lett. 123, 203201 (2019).

[51] Z. Vager, R. Naaman, and E. P. Kanter, Coulomb explosion imaging of small molecules, Science 244, 426 (1989).

[52] X. Q. Hu, Y. G. Peng, X. L. Zhu, S. C. Yan, L. Liu, W. T. Feng, D. L. Guo, Y. Gao, S. F. Zhang, D. M. Zhao, D. P. Dong, B. Hai, J. W. Xu, S. B. Zhang, X. Ma, J. G. Wang, and Y. $\mathrm{Wu}$, Breakdown of the Coulomb-explosion imaging technique induced by the ultrafast rotation of fragments, Phys. Rev. A 101, 012707 (2020).

[53] G. Galinis, C. Cacho, R. T. Chapman, A. M. Ellis, M. Lewerenz, L. G. Mendoza Luna, R. S. Minns, M. Mladenović, A. Rouzée, E. Springate, I. C. Edmond Turcu, M. J. Watkins, and K. von Haeften, Probing the Structure and Dynamics of Molecular Clusters Using Rotational Wave Packets, Phys. Rev. Lett. 113, 043004 (2014).

[54] S. Trippel, J. Wiese, T. Mullins, and J. Küpper, Communication: Strong laser alignment of solvent-solute aggregates in the gasphase, J. Chem. Phys. 148, 101103 (2018).

[55] J. D. Pickering, B. Shepperson, B. A. K. Hübschmann, F. Thorning, and H. Stapelfeldt, Alignment and Imaging of the $\mathrm{CS}_{2}$ Dimer inside Helium Nanodroplets, Phys. Rev. Lett. 120, 113202 (2018).

[56] J. Mikosch, S. Trippel, C. Eichhorn, R. Otto, U. Lourderaj, J. X. Zhang, W. L. Hase, M. Weidemüller, and R. Wester, Imaging nucleophilic substitution dynamics, Science 319, 183 (2008).

[57] Y.-P. Chang, K. Długołecki, J. Küpper, D. Rösch, D. Wild, and S. Willitsch, Specific chemical reactivities of spatially separated 3-aminophenol conformers with cold $\mathrm{Ca}^{+}$ions, Science $\mathbf{3 4 2}, 98$ (2013).

[58] E. W. Kuipers, M. G. Tenner, A. W. Kleyn, and S. Stolte, Observation of steric effects in gas-surface scattering, Nature (London) 334, 420 (1988).

[59] A. Gutiérrez-González and R. D. Beck, Unraveling the complexity of oxygen reactions on Pt surfaces, Proc. Natl. Acad. Sci. USA 116, 13727 (2019).

[60] Y. Zhang, J. P. Calupitan, T. Rojas, R. Tumbleson, G. Erbland, C. Kammerer, T. M. Ajayi, S. Wang, L. A. Curtiss, A. T. Ngo, S. E. Ulloa, G. Rapenne, and S. W. Hla, A chiral molecular propeller designed for unidirectional rotations on a surface, Nat. Commun. 10, 3742 (2019). 